\title{
Sum Rate Analysis of MU-MIMO with a 3D MIMO Base Station Exploiting Elevation Features
}

\author{
Xingwang Li, ${ }^{1}$ Lihua Li, ${ }^{2}$ Fupeng Wen, ${ }^{3}$ Junfeng Wang, ${ }^{1}$ and Chao Deng ${ }^{1}$ \\ ${ }^{1}$ College of Computer Science and Technology, Henan Polytechnic University, Jiaozuo 454000, China \\ ${ }^{2}$ State Key Laboratory of Networking and Switching Technology, Beijing University of Posts and Telecommunications, \\ Beijing 100786, China \\ ${ }^{3}$ Beijing Institute of Computer Technology and Application, Beijing 100854, China \\ Correspondence should be addressed to Lihua Li; lilihua@bupt.edu.cn
}

Received 28 August 2015; Accepted 26 November 2015

Academic Editor: Wei Xiang

Copyright (c) 2015 Xingwang Li et al. This is an open access article distributed under the Creative Commons Attribution License, which permits unrestricted use, distribution, and reproduction in any medium, provided the original work is properly cited.

Although the three-dimensional (3D) channel model considering the elevation factor has been used to analyze the performance of multiuser multiple-input multiple-output (MU-MIMO) systems, less attention is paid to the effect of the elevation variation. In this paper, we elaborate the sum rate of MU-MIMO systems with a 3D base station (BS) exploiting different elevations. To illustrate clearly, we consider a high-rise building scenario. Due to the floor height, each floor corresponds to an elevation. Therefore, we can analyze the sum rate performance for each floor and discuss its effect on the performance of the whole building. This work can be seen as the first attempt to analyze the sum rate performance for high-rise buildings in modern city and used as a reference for infrastructure.

\section{Introduction}

Currently, most research about multiuser multiple-input multiple-output (MU-MIMO) is taken with two-dimensional (2D) channel model, which only considers the horizontal dimension while ignoring the effect of elevation in the vertical dimension $[1,2]$. However, the assumption of the $2 \mathrm{D}$ propagating waves is no longer valid in the environments when the elevation spectrum is significant. Typical scenarios are indoors $[3,4]$ and in vehicles [5]. To make the channel model more applicable, several studies have taken the elevation factor into consideration [6-9]. The Wireless Initiative New Radio Project Phase II (WINNER II) releases the enhanced channel model which models the elevations of arrival and departure with parameters drawn from real channel sounding measurement [10]. Thus, WINNER II supports both indoor and outdoor scenarios, which is a significant improvement compared with the previous spatial channel model (SCM) of the 3rd Generation Partnership Project (3GPP) [11]. In [12], a simplified three-dimensional (3D) model is developed. This model assumes that the electrical wave still transmits on the $2 \mathrm{D}$ plane from the base station (BS). Only when the waves go through the scatters and arrive at the user, a significant elevation spread is present. This model is proved to perform well in indoor an in vehicle scenarios. Literature [13] proposes an approximated $3 \mathrm{D}$ antenna radiation pattern that combines the two principal cuts for azimuth (horizontal) and elevation (vertical) planes. The combination of [13] shows a tolerable approximation deviation. In [14], the 3D antenna pattern is similar to [13], but it assumes that the gains of horizontal and vertical directions are equally weighted, which makes the model more practical.

Though the elevation effect in channel modeling and performance analysis has gradually caught the researchers' attention, the exploiting of elevation variations has not yet been definitely discussed. The effect of elevation variation on communication performance is obvious in $3 \mathrm{D}$ channel model especially when the base station is close to the users and the users are distributed at different heights [14]. Nowadays, most buildings in modern cities have about twenty floors or even more. Thus, users in different floors have different elevations. Exploiting 3D MIMO at the BS covering the building enables us to make use of the distribution of users in elevation domain to improve the performance such as 


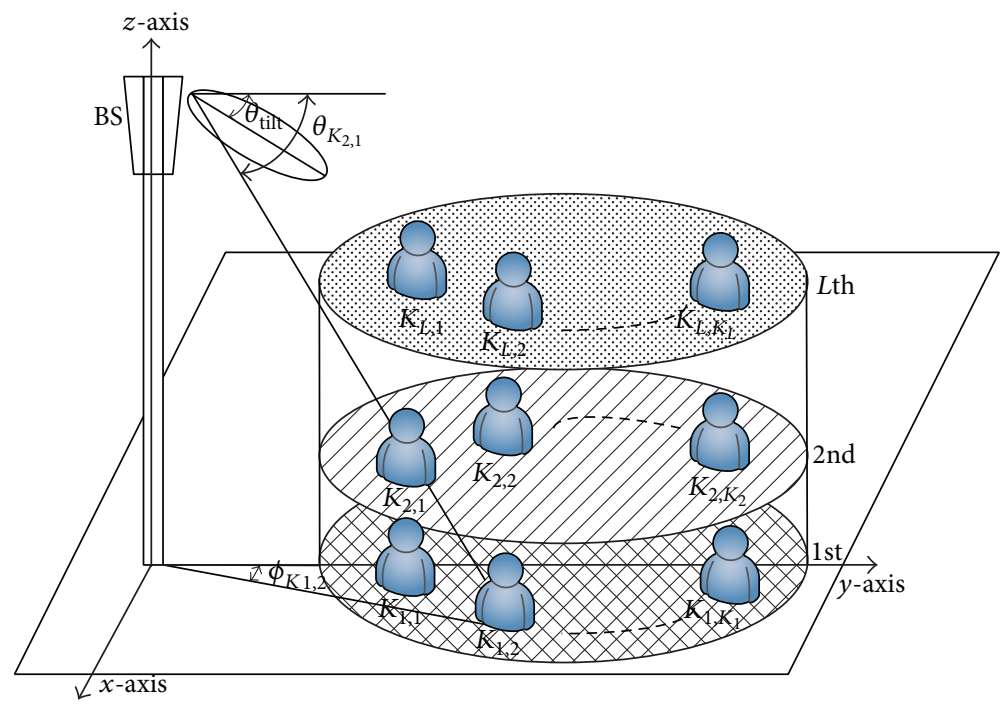

FIGURE 1: Schematic illustration of a D-MIMO system.

capacity. So how to deploy the users in the building or how to adjust the tilt angle of transmit antennas of the BS to achieve the best performance becomes a valuable problem. Several literatures like [15-19] have previously investigated the effects of user distribution on system performance. However, the common characteristic of [15-19] is that they consider user distributions in a single horizontal plane. More importantly, the $3 \mathrm{D}$ MIMO featured BS is not considered. In this paper, we mainly contribute to the sum rate derivation and analysis for uplink MU-MIMO scenario with a 3D MIMO BS considering 3D user distribution, which consists of both horizontal distribution for each floor and vertical distribution for different floors due to 3D MIMO receiving.

The contributions of this paper can be summarized as follows:

(1) We build the system model with 3D MIMO BS and introduce a building with several floors. In this paper, the deterministic sum rates of 3D MIMO system with minimum mean square error (MMSE) receivers both for the single floor and the whole building with consideration of elevation factor are deduced.

(2) For uniform user distribution, we analyze the sum rate performance of MU-MIMO system versus entire SNR with different tilt angles. It is demonstrated that the sum rate increases logarithmically with SNRs, and there is an optimal tilt angle for the sum rate.

(3) Since the radiation pattern for the antenna elements at the BS tremendously influences the radiation gain and path-loss of the user on different floors, the simulation results of the sum rate are numerically analyzed to obtain the optimal tilt angle. The optimal tilt angle can be used to adjust the antennas of BS to achieve the best performance, which is of great value.

(4) The sum rate of 3D MIMO systems for different tilt angle is investigated, which shows that elevation has a significant effects on system performance.
(5) We consider and analyze the impact of 3D user distribution in the building for different number of floors, which has very realistic significance. Since there is little research about the 3D user distribution, the result can be used as a reference for practical design.

The remainder of the paper is organized as follows. The system model and 3D MIMO channel model are presented in Section 2. Section 3 gives the derivation of the ergodic sum rate with a 3D MIMO BS exploiting variable elevation considering 3D user distribution. We present some numerical results and corresponding analysis in Section 4 before we conclude the paper in Section 5.

\section{3D MIMO System Model}

In the following, we consider an uplink single-cell MUMIMO system with a 3D MIMO BS. There are $N_{r}$ antenna elements for the 3D MIMO receiver. $K$ user terminals (UTs) each with $N_{t}$ transmit antenna elements are considered. All users are located in a building with $L$ floors. We define $K_{l, k}, l=1, \ldots, L, k=1, \ldots, K_{l}$, as the index of the $k$ th UT on $l$ th floor. The $l$ th floor has $K_{l}$ UTs which satisfies $\sum_{l=1}^{L} K_{l}=K$. A schematic illustration of the 3D MIMO system under consideration is depicted in Figure 1. In this paper, it is assumed that the BS has perfect channel state information (CSI), while all UTs have no CSI. Thus, the optimum transmission strategy is to transmit independent and equal power signal from each UT.

2.1. System Model. We now give the system model for the previously defined BS and UTs. The received signal vector $\mathbf{y} \in \mathbb{C}^{N_{r} \times 1}$ of the BS is given by

$$
\mathbf{y}=\sqrt{p_{u}} \sum_{l=1}^{L} \sum_{k=1}^{K_{l}} \mathbf{h}_{l, k} \mathbf{x}_{l, k}+\mathbf{n},
$$


where $\mathbf{x}_{l, k} \in \mathbb{C}^{N_{t} \times 1}$ is the transmitted signal vector of $\mathrm{UT}_{l, k}$, $l=1, \ldots, L, k=1, \ldots, K_{l} \cdot \mathbf{n} \in \mathbb{C}^{N_{r} \times 1}$ is the additive white Gaussion noise (AWGN) vector with zero means and unit covariance $\mathbb{E}\left[\mathbf{n n}{ }^{H}\right]=N_{0} \mathbf{I}_{N_{r}} \cdot p_{u}$ is the transmit average power, which is identical among all UTs. In our case, we have $N_{0}=1$.

Channel matrix $\mathbf{h}_{l, k}$ consists of the small-scale fading matrix $\mathbf{v}_{l, k}$ and large-scale fading matrix $\mathbf{f}_{l, k}$, which can be formulated as

$$
\mathbf{h}_{l, k}=\mathbf{f}_{l, k}^{1 / 2} \mathbf{v}_{l, k}
$$

where $\mathbf{v}_{l, k} \sim \operatorname{CN}\left(\mathbf{0}, \mathbf{I}_{N_{r}} / N_{r}\right)$. Large-scale fading matrix $\mathbf{f}_{l, k}$ is a combination of shadowing fading, path-loss, and 3D MIMO attenuation represented in [14], which can be defined as a diagonal matrix

$$
\mathbf{f}_{l, k}=f\left(x_{l, k}, y_{l, k}, z_{l, k}\right) \mathbf{I}_{N_{r}}
$$

where $f(\cdot)$ is a function to determine the variance of channel coefficient; that is, $f(\cdot)$ is the large-scale fading for UT to the BS, which models independent shadowing fading, pathloss, and 3D MIMO attenuation. We take for $f(\cdot)$ the specific model defined in the next subsection, to allow us to model antenna tilting capabilities at the BS.

2.2. 3D MIMO Channel Model. The simplified 3D channel model which appeared in $[20,21]$ is adopted in this paper, which has been engaged in 3GPP standard [22]. For simplicity, we assume that it discards explicit side lobes in favor of constant gain outside the main lobe. $G\left(\phi_{l, k}, \theta_{l, k}\right)$ is the antenna gain at the $\mathrm{BS}$ antenna array. $\phi_{l, k}$ denotes the azimuth angle measured between the direct line in the azimuth plane connecting to BS and the $y$-axis, and $\theta_{l, k}$ is the elevation angle measured between the direct line connecting $\mathrm{UT}_{l, k}$ to BS and the horizontal plane. The modeling of azimuth and elevation is done in the $3 \mathrm{D}$ coordinate system represented in Figure 1. We denote $\left(x_{\mathrm{BS}}, y_{\mathrm{BS}}, z_{\mathrm{BS}}\right)$ as the coordinate of BS, where $x_{\mathrm{BS}}, y_{\mathrm{BS}}$, and $z_{\mathrm{BS}}$ indicate the value of $x$-coordinate, $y$-coordinate, and $z$-coordinate, respectively. Similarly, we denote $\left(x_{l, k}, y_{l, k}, z_{l, k}\right)$ the coordinate value of $\mathrm{UT}_{l, k}$ in the building. For the convenience of exposition, $\Delta x_{l, k}=x_{\mathrm{BS}}-x_{l, k}$ is defined as the difference of $x$-coordinate between BS and $\mathrm{UT}_{l, k}$. In the same way, we can obtain $\Delta y_{l, k}=y_{\mathrm{BS}}-y_{l, k}$ and $\Delta z_{l, k}=z_{\mathrm{BS}}-z_{l, k}$. The distance between $\mathrm{UT}_{l, k}$ and $\mathrm{BS}$ and the corresponding azimuth and tilt angles are determined through (4)

$$
\begin{aligned}
& d_{l, k}=\sqrt{\left(\Delta x_{l, k}\right)^{2}+\left(\Delta y_{l, k}\right)^{2}+\left(\Delta z_{l, k}\right)^{2}} \\
& \phi_{l, k}=\operatorname{atan} 2\left(\Delta y_{l, k}, \Delta x_{l, k}\right) \\
& \theta_{l, k}=\operatorname{atan} 2\left(\Delta z_{l, k}, \sqrt{\left(\Delta x_{l, k}\right)^{2}+\left(\Delta y_{l, k}\right)^{2}}\right) .
\end{aligned}
$$

In order to obtain large-scale fading $f\left(x_{l, k}, y_{l, k}, z_{l, k}\right)$, antenna gain is calculated by (all " $G$-values" in decibel $(\mathrm{dB})$ )

$$
G\left(\phi_{l, k}, \theta_{l, k}\right)=G_{h}\left(\phi_{l, k}\right)+G_{v}\left(\theta_{l, k}\right)
$$

where $G_{h}\left(\phi_{l, k}\right)$ and $G_{v}\left(\theta_{l, k}\right)$ are given by

$$
\begin{aligned}
& G_{h}\left(\phi_{l, k}\right)=G_{m}-\min \left[12\left(\frac{\phi_{l, k}-\alpha_{\text {orn }}}{\mathrm{HPBW}_{h}}\right)^{2}, \mathrm{FBR}_{h}\right] \\
& G_{v}\left(\theta_{l, k}\right)=\max \left[-12\left(\frac{\theta_{l, k}-\beta_{a}}{\mathrm{HPBW}_{v}}\right)^{2}, \mathrm{SLL}_{v}\right],
\end{aligned}
$$

where $\mathrm{HPBW}_{h}$ and $\mathrm{HPBW}_{v}$ denote the half-power beamwidth in the azimuth and the elevation pattern, respectively, whereas $\mathrm{FBR}_{h}$ and $\mathrm{SLL}_{v}$ are the azimuth front-to-back ratio and the tilt side lobe level, which is relative to $G_{m} ; \alpha_{\text {orn }}$ represents the fixed orientation angle of BS array boresight relative to the $y$-axis; $\beta_{a}$ denotes the variable tilt of $\mathrm{BS}$ measured between the direct line passing through the peak of the beam and horizontal plane.

All these model parameters are obtained based on the practical antenna Kathrein 742215 [23], which is a commonly deployed antenna system and has been used in the system performance evaluation.

The path-loss consists of indoor-to-outdoor (I2O) and outdoor components which are defined according to the 3GPP standard model in [22]:

$$
\varphi_{l, k}=\frac{\varphi_{l, k}^{\mathrm{tw}} \varphi_{l, k}^{\mathrm{in}}}{d_{l, k}^{v}},
$$

where $v$ is the path-loss exponent, which is a key parameter to characterize the rate of decay of the signal power with the transceiver distance, taking values in the range of 2 (corresponding to signal propagation in free space) to 6 . Typical values for the path-loss are 4 for an urban macrocell environment and 3 for urban microcell environment [24]. $\varphi_{l, k}^{\mathrm{tw}}$ and $\varphi_{l, k}^{\mathrm{in}}$ are the wall penetration loss and the indoor propagation loss, respectively, whose values are determined by [22].

For shadowing fading, the log-normal shadowing fading model is adopted, which has been the prevalent model in the characterization of shadowing effects in wireless and satellite communications environments [25]. Thus, the probability density function (PDF) of shadowing fading coefficient is

$$
\begin{array}{r}
p\left(\xi_{l, k}\right)=\frac{\eta}{\xi_{l, k} \sqrt{2 \pi \sigma_{l, k}^{2}}} \exp \left(-\frac{\left(\eta \ln \left(\xi_{l, k}\right)-\mu_{l, k}\right)^{2}}{2 \sigma_{l, k}^{2}}\right), \\
\xi_{l, k} \geq 0,
\end{array}
$$

where $\eta=10 / \ln 10$, while $\mu_{l, k}(\mathrm{~dB})$ and $\sigma_{l, k}(\mathrm{~dB})$ are the mean and standard deviation of the random variable $(\mathrm{RV}) \ln \left(\xi_{l, k}\right)$.

Motivated by the previous discussion, we can conclude that large-scale fading function $f\left(x_{l, k}, y_{l, k}, z_{l, k}\right)$ is composed of shadowing fading, path-loss, and 3D antenna attenuation. Thus, overall loss factor contained in $\mathbf{f}_{l, k}$ is

$$
f\left(x_{l, k}, y_{l, k}, z_{l, k}\right)=\xi_{l, k} \varphi_{l, k} 10^{G\left(\phi_{l, k}, \theta_{l, k}\right) / 10} .
$$




\section{Acheivable Sum Rate for 3D MIMO}

3.1. Sum Rate for 3D MIMO. In the following, we focus on the ergodic sum rate of $3 \mathrm{D}$ MIMO MMSE receivers. The equalization output of $\mathrm{UT}_{l, k}$ is given by

$$
\widetilde{\mathbf{x}}_{l, k}=\mathbf{g}_{l, k}^{H} \mathbf{y} \stackrel{(a)}{=} \mathbf{g}_{l, k}^{H} \mathbf{h}_{l, k} \mathbf{x}_{l, k}+\sum_{m=1}^{L} \sum_{n \neq k}^{K_{m}} \mathbf{g}_{l, k}^{H} \mathbf{h}_{m, n} \mathbf{x}_{m, n}+\mathbf{g}_{l, k}^{H} \mathbf{n},
$$

where $\mathbf{g}_{l, k}$ is the detection matrix for $\mathrm{UT}_{l, k}$ and $(a)$ is derived from (1). Thus, the equalization output consists of two components: (I) the desired signal component $\mathbf{g}_{l, k}^{H} \mathbf{h}_{l, k} \mathbf{x}_{l, k}$ and (II) the interference-plus-noise component $\sum_{m=1}^{L} \sum_{n \neq k}^{K_{m}} \mathbf{g}_{l, k}^{H} \mathbf{h}_{m, n} \mathbf{x}_{m, n}+\mathbf{g}_{l, k}^{H} \mathbf{n}$. The instantaneous received signal-to-interference-plus-noise (SINR) of $\mathrm{UT}_{l, k}$ can be expressed as

$$
\gamma_{l, k}^{u l}=\frac{p_{u}\left\|\mathbf{g}_{l, k}^{H} \mathbf{h}_{l, k}\right\|^{2}}{\mathbf{g}_{l, k}^{H}\left(p_{u} \sum_{m=1}^{L} \sum_{n \neq k}^{K_{m}} \mathbf{h}_{m, n} \mathbf{h}_{m, n}^{H}+\mathbf{I}_{N_{r}}\right) \mathbf{g}_{l, k}},
$$

where $\mathbf{g}_{l, k}$ is the MMSE matched filter, which is represented as

$$
g_{l, k}=\left(\sum_{m=1}^{L} \sum_{n=1}^{K_{m}} \mathbf{h}_{m, n} \mathbf{h}_{m, n}^{H}+\frac{\mathbf{I}_{N_{r}}}{p_{u}}\right)^{-1} \mathbf{h}_{l, k} .
$$

Combining (7) with (10), 3D MIMO channel matrix $\mathbf{h}_{l, k}$ and 3D MIMO MMSE filter can be expressed as

$$
\begin{aligned}
& \mathbf{h}_{l, k}=\left(f \mathbf{I}_{N r}\right)^{1 / 2} \mathbf{v}_{l, k}=\left(\xi_{l, k} \varphi_{l, k} 10^{G\left(\phi_{l, k}, \theta_{l, k}\right) / 10} d_{l, k}^{-v}\right)^{1 / 2} \mathbf{v}_{l, k} \\
& \mathbf{g}_{l, k}=\left(\sum_{m=1}^{L} \sum_{n=1}^{K_{m}}\left(\frac{\xi_{m, n} \varphi_{m, n} 10^{G\left(\phi_{m, n}, \theta_{m, n}\right) / 10}}{d_{m, n}^{v}}\right) \mathbf{v}_{m, n} \mathbf{v}_{m, n}^{H}\right. \\
&+\left.\frac{\mathbf{I}_{N_{r}}}{p_{u}}\right)^{-1} \times\left(\xi_{m, n} \varphi_{m, n} 10^{G\left(\phi_{m, n}, \theta_{m, n}\right) / 10} d_{l, k}^{-v}\right)^{1 / 2} \mathbf{v}_{l, k} \\
& \stackrel{(b)}{=}\left(\frac{1}{N_{r}} \sum_{m=1}^{L} \sum_{n=1}^{K_{m}}\left(\xi_{m, n} \varphi_{m, n} 10^{G\left(\phi_{m, n}, \theta_{m, n}\right) / 10} d_{m, n}^{-v}\right)\right. \\
&\left.+\frac{1}{p_{u}}\right)^{-1} \times\left(\xi_{m, n} \varphi_{m, n} 10^{G\left(\phi_{m, n}, \theta_{m, n}\right) / 10} d_{l, k}^{-v}\right)^{1 / 2} \mathbf{v}_{l, k},
\end{aligned}
$$

where $(b)$ is obtained directly from $\mathbf{v}_{l, k} \sim \mathrm{CN}\left(\mathbf{0}, \mathbf{I}_{N r} / N_{r}\right)$.

Substituting (13) into (11), the output SINR for $\mathrm{UT}_{l, k}$ is further represented as

$$
\begin{aligned}
\gamma_{l, k}^{u l} & =\left(\xi_{l, k} \varphi_{l, k} 10^{G\left(\phi_{l, k}, \theta_{l, k}\right) / 10} d_{l, k}^{-v}\right) \\
\cdot & \mathbf{v}_{l, k}\left(\sum_{m=1}^{L} \sum_{n \neq 1}^{K_{m}}\left(\xi_{m, n} \varphi_{m, n} 10^{G\left(\phi_{m, n}, \theta_{m, n}\right) / 10} d_{m, n}^{-v}\right) \mathbf{v}_{m, n} \mathbf{v}_{m, n}^{H}\right.
\end{aligned}
$$

$$
\begin{aligned}
& \left.+\frac{\mathbf{I}_{N_{r}}}{p_{u}}\right)^{-1} \mathbf{v}_{l, k}^{H}=\frac{N_{r}}{p_{u}}\left(\xi_{m, n} \varphi_{m, n} \frac{10^{G\left(\phi_{l, k}, \theta_{l, k}\right) / 10}}{N_{r} d_{l, k}^{v}}\right) \\
& \cdot\left(\sum_{m=1}^{L} \sum_{n \neq 1}^{K_{m}}\left(\xi_{m, n} \varphi_{m, n} 10^{G\left(\phi_{m, n}, \theta_{m, n}\right) / 10} d_{m, n}^{-v}\right)+\frac{1}{p_{u}}\right)^{-1} \\
& \cdot \mathbf{I}_{N_{t}} \cdot
\end{aligned}
$$

As discussed in Section 2, we assume that overall receivers have sufficient CSI and UTs location information. The local information is acquired easily by global positioning system (GPS) or other positioning technologies. Thus, BS can perform MMSE detection to maximize the SINR. The achievable ergodic sum rate is given by

$$
R_{\mathrm{sum}}^{u l}=\sum_{l=1}^{L} \sum_{k=1}^{K_{l}} \mathbb{E}\left(\log _{2}\left(\operatorname{det}\left(\mathbf{I}_{N_{t}}+\gamma_{l, k}^{u l}\right)\right)\right),
$$

where the expectation is taken since channel is assumed to be ergodic, which means that a reasonably long time sample of channel (fading) realizations has a distribution similar to the statistical distribution of the channel.

3.2. User Distribution. In practice, the performance of MIMO systems is affected not only by the fading but also by the user distributions [15-19]. In the following, we consider the spatial user distribution in the building, which consists of both horizontal plane for each floor and the vertical plane for users in different floors. It is assumed that all floors of the building model are circles and have the same radius $R$.

3.2.1. Horizontal User Distribution. For horizontal plane, uniform distribution, Gaussian distribution, and linear are considered. For the first case, we assume all users (desired and interfering users) are independently and uniformly distributed on the circular floor. The typical cases are dormitories and residential buildings. The PDF of uniform distribution is represented by

$$
f_{u}(x)=\frac{2 x}{R^{2}}, \quad|x| \leq R,
$$

where, following the PDF property, it is not difficult to compute $U$ as $1 / 2 R$.

For the second case, most users are concentrated in the center of the floor and the density of users along the radius tends to be a Gaussian curve. Typical scenes are "hot-spots" such as city centers, shopping malls, and office areas. The PDF of Gaussian distribution is represented by

$$
f_{\mathrm{Ga}}(x)=\left(\frac{G}{\sigma \sqrt{2 \pi}}\right) e^{-x^{2} / 2 \sigma^{2}}, \quad|x| \leq R,
$$

where $G$ and $\sigma$ are constants. Applying the properties of PDF, Gaussian distribution, and probability integral, we obtain $G=2 \Phi(3 / \sqrt{2})^{-1}, \sigma=R / 3$, where $\Phi(u)=\operatorname{erf}(u)=$ $(\sqrt{\pi} / 2) \int_{0}^{u} e^{-x^{2}} d x$ being the error function [26, Eq. (8.250.1)]. 
TABLE 1: Systme parameters.

\begin{tabular}{|c|c|c|}
\hline Parameter & Details & Value \\
\hline$L$ & Number of floors & 3 \\
\hline$K$ & Number of UTs & 24 \\
\hline$N_{r}$ & Number of BS antennae & 50 \\
\hline$N_{t}$ & Number of UT antennae & 2 \\
\hline$D$ & Distance between BS and the center of the building & $200 \mathrm{~m} / 1000 \mathrm{~m}$ \\
\hline$R_{B}$ & Radius of building & $100 \mathrm{~m}$ \\
\hline$h_{\mathrm{BS}}$ & Height of BS & $30 \mathrm{~m}$ \\
\hline$h_{\mathrm{UT}}$ & Height of UT & $1.5 \mathrm{~m}$ \\
\hline$h_{\text {floor }}$ & Height of floor & $5 \mathrm{~m}$ \\
\hline$\mu$ & Shadowing fading mean & $4 \mathrm{~dB}$ \\
\hline$\sigma$ & Shadowing fading standard deviation & $4 \mathrm{~dB}$ \\
\hline$v$ & Path-loss exponent & 4 \\
\hline$\varphi^{\mathrm{tw}}$ & Wall penetration loss & $0.01(-20 \mathrm{~dB})$ \\
\hline$\varphi^{\text {in }}$ & Inside loss & $0.5 d_{2 \mathrm{D} \_ \text {in }^{*}}{ }^{*}$ \\
\hline$A_{\max }$ & Maximum antenna gain & $18 \mathrm{dBi}$ \\
\hline $\mathrm{HPBW}_{h}$ & Half-power beam-width in the azimuth pattern & $65^{\circ}$ \\
\hline $\mathrm{HPBW}_{v}$ & Half-power beam-width in the elevation pattern & $6.5^{\circ}$ \\
\hline FBR $h$ & Azimuth front-to-back ratio & $30 \mathrm{~dB}$ \\
\hline $\mathrm{SLL}_{v}$ & Tilt side love level & $-18 \mathrm{~dB}$ \\
\hline$\alpha_{\text {orn }}$ & Fixed orientation angle & $0^{\circ}$ \\
\hline
\end{tabular}

As for the last case, users are distributed in the floor and the density of users along the radius tends to be a linear curve. The PDF of linear distribution is represented by

$$
f_{\text {lin }}(x)=-A|x|+B, \quad|x| \leq R,
$$

where $A$ and $B$ are the slope and intercept, separately. Capitalizing the property of PDF, it is not difficult to calculate the value of $A$ and $B$ as $1 / 2 R^{2}$ and $1 / 2 R$, respectively.

3.2.2. Vertical User Distributions. Generally in a $L$-floor building, the number of users on each floor is variant, because most "hot-spots" such as shopping malls and supermarkets are always on the first floor (especially in China). So we assume that a higher concentration of users is distributed on the lower floors while lower concentration of users on the higher floors. We define the user distribution in the vertical dimension as the distribution of the number of users on each floor whose probability mass functions (PMF) are given by

$$
p(l)= \begin{cases}N Y^{l} & l=1, \ldots, L \\ 0 & \text { others }\end{cases}
$$

where $l$ represents the number of floor in the building and $\Upsilon^{l}$ denotes the ratio between the number of users on the $l$ th floor and the whole building. $N$ is a constant to satisfy that the sum of all users of all floors in the building is $K$ :

$$
K=\sum_{l=1}^{L} N \Upsilon^{l}
$$

The purpose of the paper is to derive the sum rate of $3 \mathrm{D}$ MIMO and analyze the impact of user distributions on the sum rate in building with respect to variable BS tilt angle. In particular, we investigate the optimal tilt angle to maximize the performance of the building and each floor. This is very interesting in practice scenario, which can be used as a reference for infrastructure.

\section{Numerical Results}

4.1. Simulation Assumptions. We investigate the sum rate of different user distribution schemes using Monte Carlo simulation. In the simulation, the simulation parameter settings are given in Table 1.

For all the following simulation, we consider two configurations with $D=200 \mathrm{~m}$ and $D=1000 \mathrm{~m}$, so the coordinates of BS and the center of three floors of the building are $(0,0,30),(200,0,0),(200,0,5)$, and $(200,0,10)$ and $(1000,0,0),(1000,0,5)$, and $(1000,0,10)$, respectively.

In the following, we investigate the performance of four different schemes as follows:

(1) For uniform user distribution, we assess the sum rate against the SNR for different tilt angle.

(2) The sum rate corresponding to uniform, normal (Gaussian), and linear distribution with 3D MIMO is provided.

(3) The sum rate of each floor for the three user distributions with the same parameter configuration as in (1) is analyzed. 


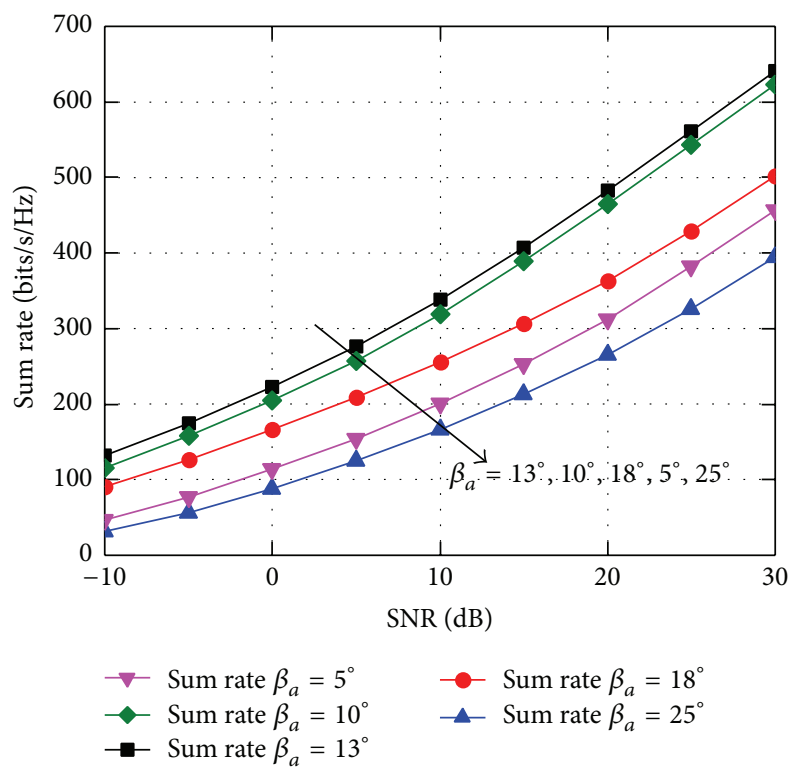

FIGURE 2: Simulated sum rate against the SNR for uniform distribution $\left(N_{r}=50, N_{t}=2, L=3, K=24, D=200 \mathrm{~m}, R=100 \mathrm{~m}, v=4\right.$, and $\beta_{a}=5^{\circ}, 10^{\circ}, 13^{\circ}, 18^{\circ}, 25^{\circ}$ ).

(4) The impact of vertical user distribution on the sum rate for different floors is shown.

Since $R=100 \mathrm{~m}$, the parameters are calculated as $U=5 \times$ $10^{-3}, \sigma=100 / 3, G=1.2 \times 10^{-2}, g=1.33 \times 10^{-4}, A=5 \times 10^{-3}$, and $B=5 \times 10^{-5}$. For $D=200 \mathrm{~m}$ and $D=1000 \mathrm{~m}$, the range of the relative angle between the direct line from UTs to BS and horizontal plane is $1.92^{\circ}<\theta<16.70^{\circ}$ and $1.04^{\circ}<\theta<1.91^{\circ}$.

4.2. Sum Rate versus SNR. We first analyze the performance of the sum rate against different average SNR for tilt angle $\beta_{a}=5^{\circ}, 10^{\circ}, 13^{\circ}, 18^{\circ}, 25^{\circ}$. In Figure 2, it can be observed that the sum rate increases logarithmically with the average SNR for these tilt angles. In addition, the sum rate increases with the tilt angle before the tilt angle reaches the critical tilt angle and then decreases with the further increase.

4.3. Optimal Tilting for Sum Rate. In terms of system performance in Figure 3, we observe that there is an optimal tilt angle that corresponds to the optimal performance. The smaller tilt angle yields lower sum rate due to the smaller antenna gain effects. For larger tilt angle, users also experience lower antenna gain due to deviating the optimal tilt angle. Thus, the sum rate dramatically drops. As seen from Figure 2, there is a global trend for all the three user distributions that if we increase or decrease the tilt angle from optimal tilt angle, the sum rate will decrease. In Figure 3, we also see that sum rate of uniform distribution is inferiors to normal distribution and linear distribution. This coincides with the results of [14], which is due to a trade-off between path-loss and optimal tilt effects.

Figure 4 shows the sum rate results with the distance parameter setting $D=1000 \mathrm{~m}$. For the three user distributions, the performances are similar due to the very small

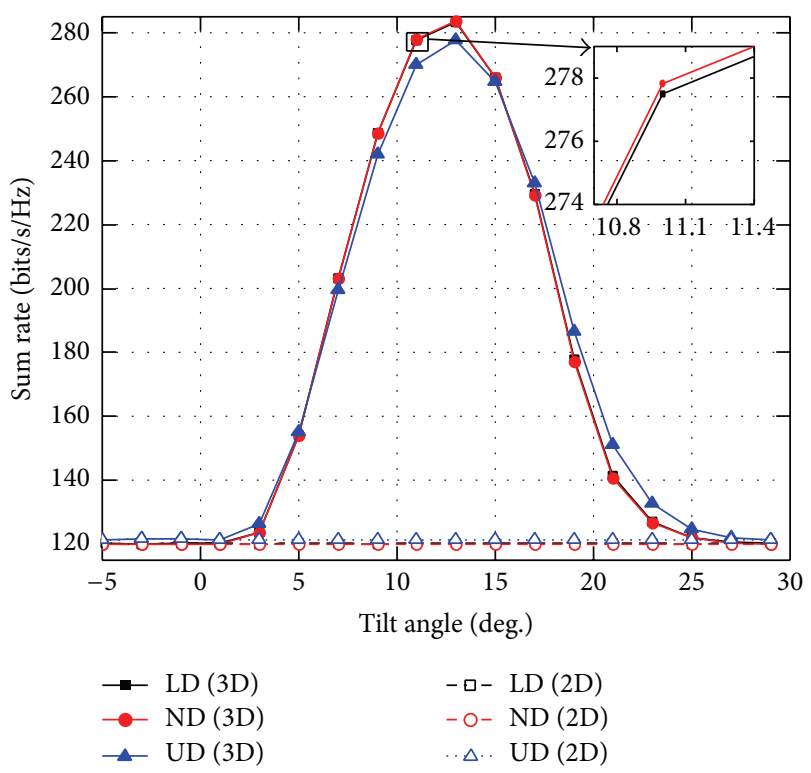

FIGURE 3: Simulated sum rate against the tilt angle for three user distributions $\left(p_{u}=5 \mathrm{~dB}, N_{r}=50, N_{t}=2, L=3, K=24, D=\right.$ $200 \mathrm{~m}, R=100 \mathrm{~m}, v=4$, UD: uniform distribution, ND: normal distribution, and LD: linear distribution).

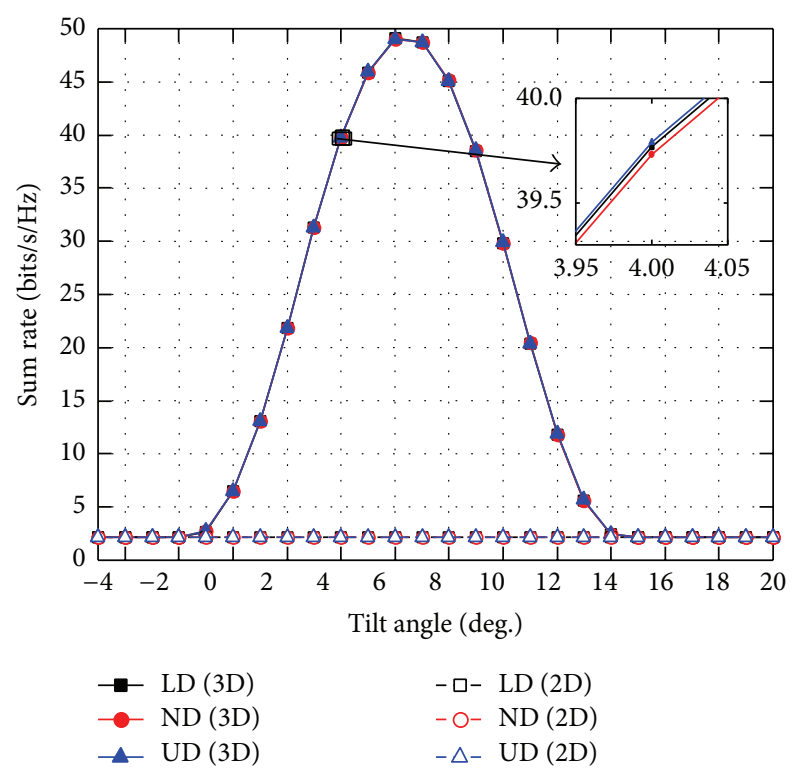

FIGURE 4: Simulated sum rate against tilt angle for three user distributions $\left(p_{u}=5 \mathrm{~dB}, N_{r}=50, N_{t}=2, L=3, K=24\right.$, $D=1000 \mathrm{~m}, R=100 \mathrm{~m}, v=4$, UD: uniform distribution, ND: normal distribution, and LD: linear distribution).

angular difference and the high path-loss. This shows that, for large distance setting, the effects of user distributions on the sum rate are quite small and can be ignored due to distances between BS and UTs.

From Figures 3 and 4, some conclusions can be drawn. First, the globe trends for the two figures are similar because 


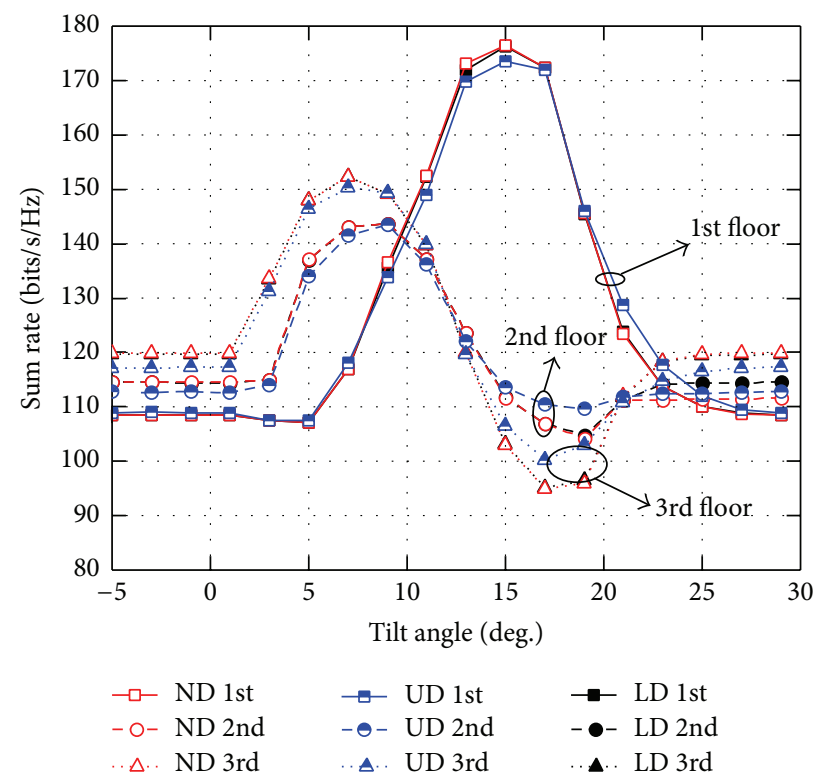

FIGURE 5: Simulated sum rate against tilt angle for three user distributions $\left(p_{u}=5 \mathrm{~dB}, N_{r}=50, N_{t}=2, L=3, K=24, D=\right.$ $200 \mathrm{~m}, v=4$, UD: uniform distribution, ND: normal distribution, and LD: linear distribution).

of the same trends for both antenna gain and path-loss. Second, the uniform user distribution has a better performance than the normal and linear one due to a trade-off between path-loss and tilt angle effect. Third, the latter figure yields small range of tilt angle with small sum rate since the pathloss is large while the angular variation is smaller. Besides, the effect of user distributions is more significant in Figure 3 than that in Figure 4.

4.4. Performance for Each Floor. In this part, we focus on the influence of the optimal tilt angle and distance between BS and UTs on the sum rate for each floor. To this end, we investigate the sum rate of each floor for the three user distributions with the same parameters as in Section 4.2, and then we investigate the impact of the tilt angle. For $D=200 \mathrm{~m}$ in Figure 5, it can be seen that the optimal tilt angles of the three user distributions are quite similar while the optimal tilt angle for a single floor is little different. In addition, appropriate user distribution can improve the sum rate. These two results are very useful in reality to maximize the performance of systems. For $D=1000 \mathrm{~m}$ in Figure 6, this is a more interesting case that the influence of user distribution on the sum rate can be ignored due to the higher path-loss. Therefore, tilt angle is the key factor to improve the performance of the system.

In Figures 5 and 6, there exists the same phenomenon that the first floor has a larger tilt angle than the second and the third one for the three distributions due to the largest distances. Thus, first floor has the largest optimal angle and the second floor has an intermediate optimal angle while the third floor has the smallest optimal angle. It is of practical significance to select an appropriate tilt angle to maximize

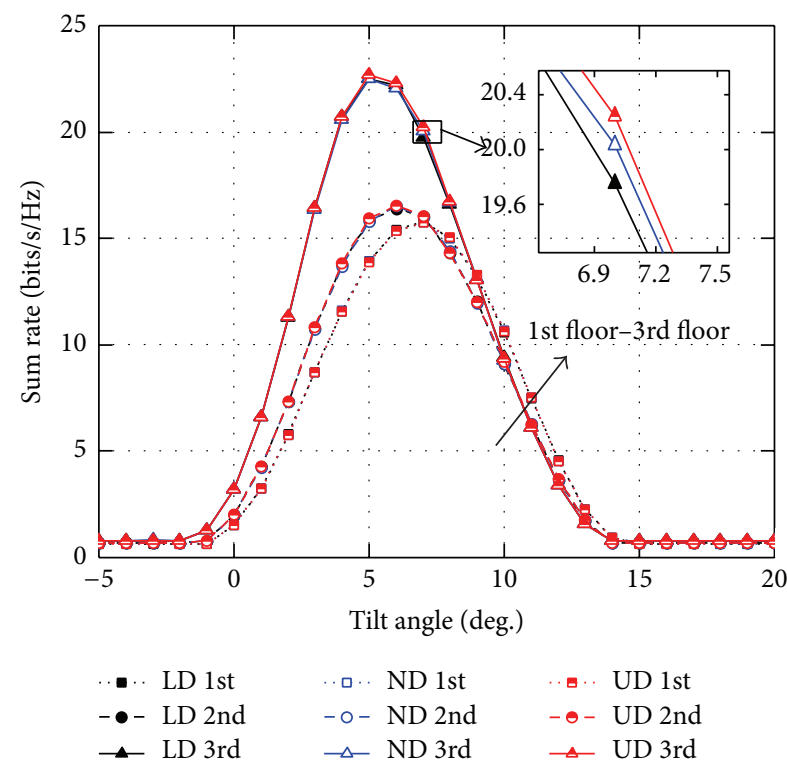

FIGURE 6: Simulated sum rate against the tilt angle of each floor for three user distributions $\left(p_{u}=5 \mathrm{~dB}, N_{r}=50, N_{t}=2, L=3, K=24\right.$, $D=1000 \mathrm{~m}, v=4, L=3, K=24, D=1000 \mathrm{~m}, v=4, R=100 \mathrm{~m}$, UD: uniform distribution, ND: normal distribution, and LD: linear distribution).

the communication performance for the whole building and each floor.

4.5. Vertical User Distributions. We now study the impact of vertical user distribution on the sum rate for $L=1$ and $L=3$. Related parameters are similar to Figure 4, except that the total UT number is $K=42$. We consider that linear vertical distribution with the slope is $Y=1 / 2$, which means that number in the $l$ th floor is twice than that of the $(l+1)$ th floor. For $L=1$, there is only one floor and all UTs are located on the floor. For $L=3$, the number of users on the first floor is 24 , and the numbers of UTs on the second and third floors are 12 and 6 , respectively.

The sum rate considering vertical user distribution is illustrated in Figures 7 and 8. The two figures show similar global trends as Figures 3 and 4 for all tilts angles and floors. However, the optimal angle of Figure 7 is larger than that of Figure 3 due to the fact that the sum rate is dominated by the first floor, which has the most UTs and the largest optimal angle than other floors. For the same reason, we can observe the same phenomenon for normal and linear user distributions. In addition, we can find that the sum rates of linear and normal are almost the same when considering the vertical distribution due to the similar PDF curve. Corresponding reason is that the sums of UTs on the first and second floors for these two distributions are nearly the same, which determines the optimal angles. When we only consider the horizontal distribution, the sum rate of linear distribution is smaller than that of normal distribution. Moreover, when the tilt angle reaches critical angle, the sum rate of case $L=1$ is larger than that of case $L=3$, otherwise the sum rate of case $L=3$ is larger than that of case $L=1$. 


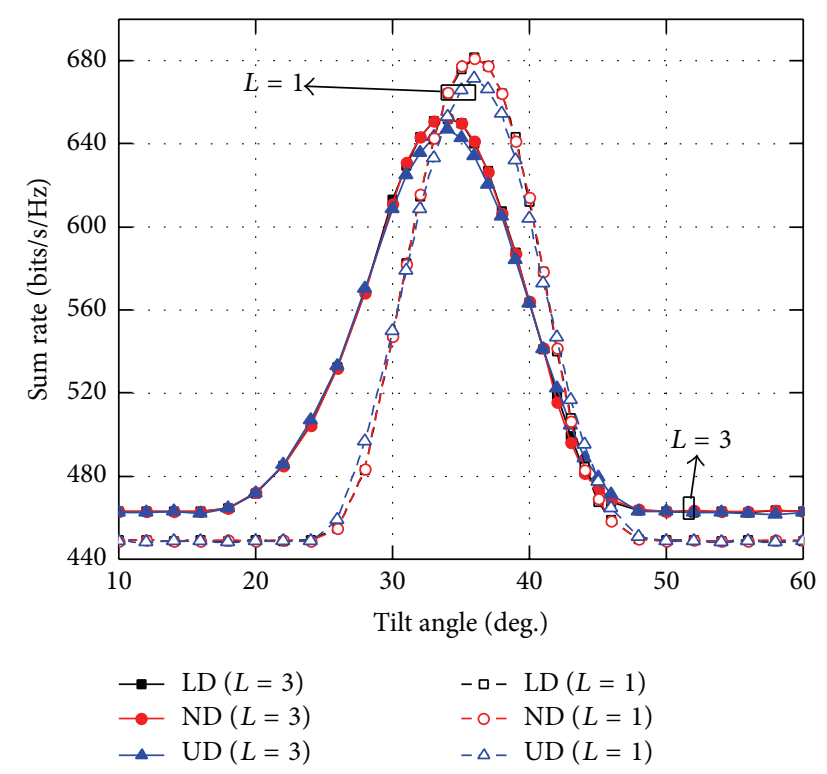

FIgURE 7: Simulated sum rate against the tilt angle for three user distributions $\left(p_{u}=5 \mathrm{~dB}, N_{r}=100, N_{t}=1, L=1,3, K=42, D=\right.$ $200 \mathrm{~m}, R=100 \mathrm{~m}, v=4$, UD: uniform distribution, ND: normal distribution, and LD: linear distribution).

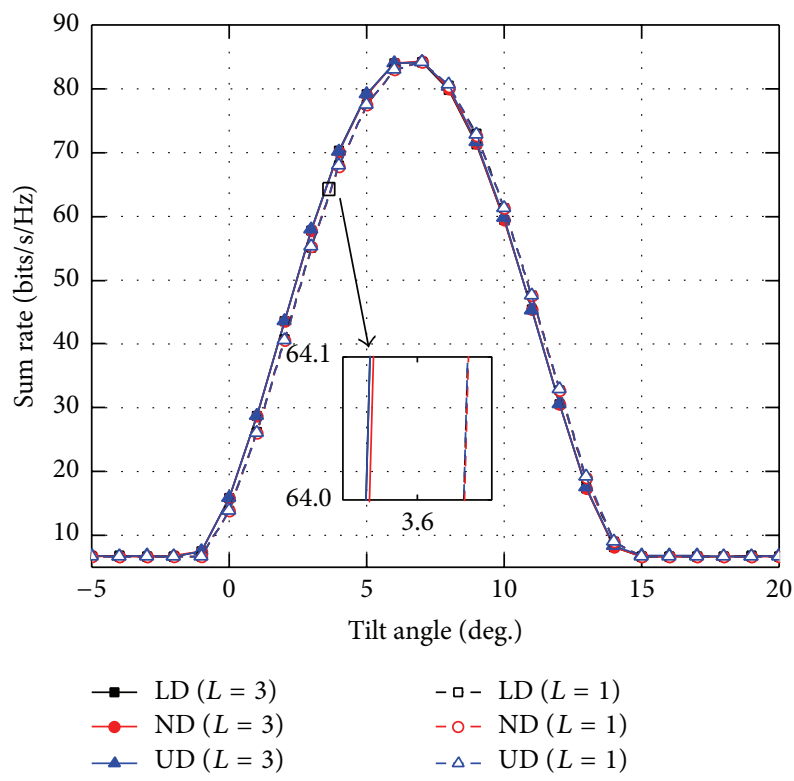

FIGURE 8: Simulated sum rate against the tilt angle for three user distributions $\left(p_{u}=5 \mathrm{~dB}, N_{r}=50, N_{t}=1, L=1,3, K=42\right.$, $D=1000 \mathrm{~m}, R=100 \mathrm{~m}, v=4$, UD: uniform distribution, ND: normal distribution, and LD: linear distribution).

So we can conclude that appropriate vertical distribution can be adopted in order to obtain higher performance, while the optimal angle of Figure 8 is quite similar to Figure 4 due to the effect of large high path-loss and small angle variation. In addition, there are almost no differences in sum rate between cases $L=1$ and $L=3$. This shows that, for large distance setting, the effects of user distributions on sum rate are quite small and can be ignored.

\section{Conclusion}

With 3D BS exploiting elevation features, we deduce the exact analytical expression of the sum rate for single cell MUMIMO uplink system. The impacts of antenna tilt angle on the sum rate for both the whole building and the single floor are investigated. We find that appropriate tilt angle can compensate for the sum rate gain lost by the path-loss. Therefore, this paper can be used to analyze and optimize the performance. Furthermore, the functions of each floor and the related user distributions can be designed with reference to these results. This can be an important topic in the future design for wireless system.

\section{Conflict of Interests}

The authors declare that there is no conflict of interests regarding the publication of this paper.

\section{Acknowledgments}

This work was supported by the National 863 Project (no. 2014AA01A705), the National Science and Technology Major Project (no. 2015ZX03001034), the Doctoral Scientific Funds of Henan polytechnic University (no. 60907013), the National Natural Science Foundation of China (Grant no. 61501404), Fundamental and Advanced Research Project of Henan Province of China under Grant (no. 132300410461), and the Fundamental Research Funds for the Universities of Henan Province. (no. NSFRF140125).

\section{References}

[1] K. Zheng, F. Liu, L. Lei, C. Lin, and Y. Jiang, "Stochastic performance analysis of a wireless finite-state Markov channel," IEEE Transactions on Wireless Communications, vol. 12, no. 2, pp. 782-793, 2013.

[2] K. Zheng, L. Zhao, J. Mei, M. Dohler, W. Xiang, and Y. Peng, "10 $\mathrm{Gb} / \mathrm{s}$ hetsnets with millimeter-wave communications: access and networking_challenges and protocols," IEEE Communications Magazine, vol. 53, no. 1, pp. 222-231, 2015.

[3] X. Li, L. Li, L. Xie, X. Su, and P. Zhang, "Performance analysis of 3D massive MIMO cellular systems with collaborative base station," International Journal of Antennas and Propagation, vol. 2014, Article ID 614061, 12 pages, 2014.

[4] L. Xie, L. Li, and X. Li, "Sum rate analysis of Multicell MuMIMO with 3D user distribution and base station tilting," in Proceedings of the IEEE Vehicular Technology Conference (VTC Fall '14), pp. 1-6, September 2014.

[5] C.-X. Wang, X. Cheng, and D. I. Laurenson, "Vehicle-to-vehicle channel modeling and measurements: recent advances and future challenges," IEEE Communications Magazine, vol. 47, no. 11, pp. 96-103, 2009.

[6] K. Zheng, L. Zhao, J. Mei, B. Shao, W. Xiang, and L. Hanzo, "Survey of large-scale MIMO systems," IEEE Communications Surveys \& Tutorials, vol. 17, no. 3, pp. 1738-1760, 2015.

[7] A. Kammoun, H. Khanfir, Z. Altman, M. Debbah, and M. Kamoun, "Preliminary results on 3D channel modeling: from theory to standardization," IEEE Journal on Selected Areas in Communications, vol. 32, no. 6, pp. 1219-1229, 2014. 
[8] K. Zheng, S. L. Ou, and X. F. Yin, "Massive MIMO channel models: a survey," International Journal of Antennas and Propagation, vol. 2014, Article ID 848071, 10 pages, 2014.

[9] Q. Nadeem, A. Kammoun, M. Debbah, and M. Alouini, “3D massive MIMO systems: modeling and performance analysis," IEEE Transactions on Wireless Communications, vol. 14, no. 12, pp. 6926-6939, 2015.

[10] A. Osseiran, E. Hardouin, A. Gouraud, M. Boldi, and I. Cosvic, "The road to IMT-advanced communication systems: stateof-the art and innovation areas addressed by the WINNER+ project," IEEE Communications Magazine, vol. 47, no. 6, pp. 3847, 2009.

[11] M. Narandzic, C. Schneider, R. Thoma, T. Jamsa, P. Kyosti, and X. Zhao, "Comparison of SCM, SCME, and WINNER channel models," in Proceedings of the IEEE 65th Vehicular Technology Conference (VTC Spring '07), pp. 413-417, Dublin, Ireland, April 2007.

[12] M. Shafi, M. Zhang, A. F. M. A. L. Moustakas et al., "Polarized MIMO channels in 3-D: models, measurements and mutual information," IEEE Journal on Selected Areas in Communications, vol. 24, no. 3, pp. 514-527, 2006.

[13] T. G. Vasiliadis, A. G. Dimitriou, and G. D. Sergiadis, "A novel technique for the approximation of 3-D antenna radiation patterns," IEEE Transactions on Antennas and Propagation, vol. 53, no. 7, pp. 2212-2219, 2005.

[14] X. Li, L. Li, and L. Xie, "Achievable sum rate analysis of ZF receivers in 3D MIMO systems," KSII Transactions on Internet and Information Systems, vol. 8, no. 4, pp. 1368-1389, 2014.

[15] K. S. Gilhousen, I. M. Jacobs, R. Padovani, A. J. Viterbi, L. A. Weaver Jr., and C. E. Wheatley III, "On the capacity of a cellular CDMA system," IEEE Transactions on Vehicular Technology, vol. 40, no. 2, pp. 303-312, 1991.

[16] K. Kim and I. Koo, CDMA Systems Capacity Engineering, Artech House, Norwood, Mass, USA, 2005.

[17] K. B. Baltzis, "Hexagonal vs circular cell shape: a comparative analysis and evaluation of the two popular modeling approximations," in Cellular Networks-Positioning, Performance Analysis, Reliability, A. Melikov, Ed., chapter 4, InTech, Rijeka, Croatia, 2011.

[18] G. Rajamani and J. Kuriacose, "Effect of non-uniform traffic distributions on performance of a cellular CDMA system," in Proceedings of the IEEE 6th International Conference on Universal Personal Communications Record, 1997. Conference Record, vol. 2, pp. 598-602, San Diego, Calif, USA, October 1997.

[19] J. S. Thompson, P. M. Grant, and B. Mulgrew, "Effects of user distribution on CDMA antenna array receivers," in Proceedings of the 1st IEEE Signal Processing Workshop on Signal Processing Advances in Wireless Communications (SPAWC'97), pp. 181-184, April 1997.

[20] A. Muller, J. Hoydis, R. Couillet, and M. Cdebbah, "Optimal 3D cell planning: a random matrix approach," in Proceedings of the IEEE Global Communications Conference (GLOBECOM '12), pp. 4512-4517, IEEE, Anaheim, Calif, USA, December 2012.

[21] F. Gunnarsson, M. N. Johansson, A. Furuskär et al., "Downtilted base station antennas-a simulation model proposal and impact on HSPA and LTE performance," in Proceedings of the 68th Semi-Annual IEEE Vehicular Technology (VTC '08), pp. 15, Calgary, Canada, September 2008.

[22] 3GPP, “3D channel model for LTE”, 3GPP TR 36.873 V12.0.0, 2014.

[23] Kathrein Scala Division 742215V01, http://www.kathrein-scala .com/catalog/742215V01_2015-12-15.pdf.
[24] G. L. Stüber, Principles of Mobile Communication, Springer, New York, NY, USA, 3rd edition, 2012.

[25] M. K. Simon and M. Alouini, Digital Communication over Fading Channel, John Wiley \& Sons, New York, NY, USA, 2nd edition, 2005.

[26] I. S. Gradshteyn and I. M. Ryzhik, Table of Intergrals, Series, and Products, Academic Press, San Diego, Calif, USA, 7th edition, 2007. 

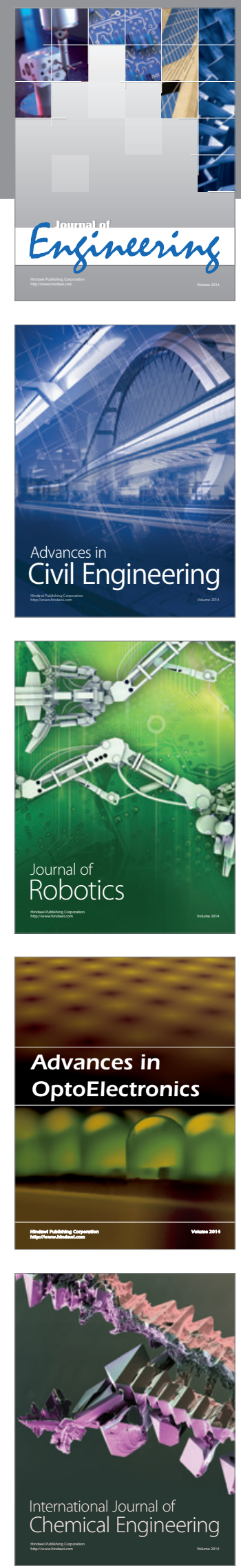

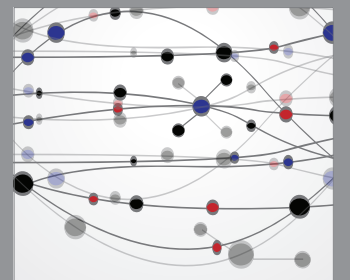

The Scientific World Journal
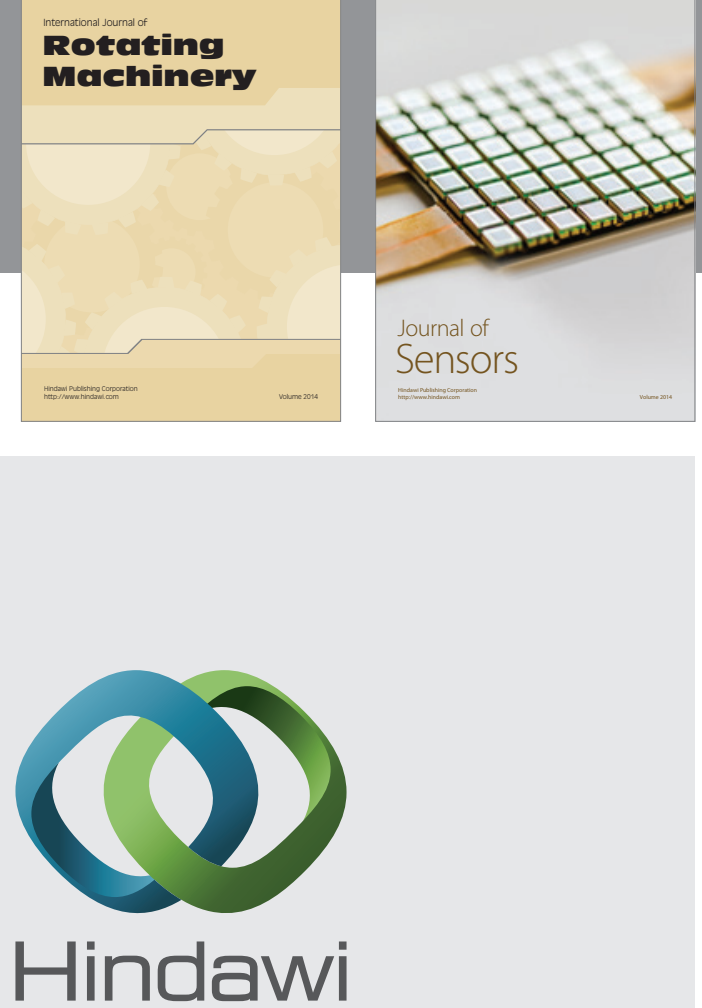

Submit your manuscripts at http://www.hindawi.com
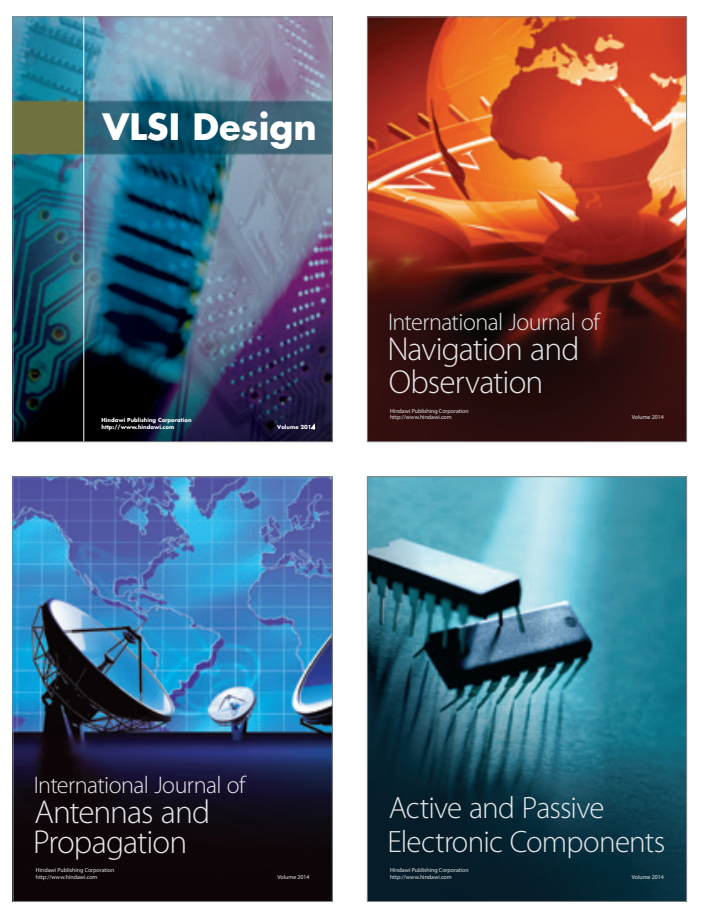
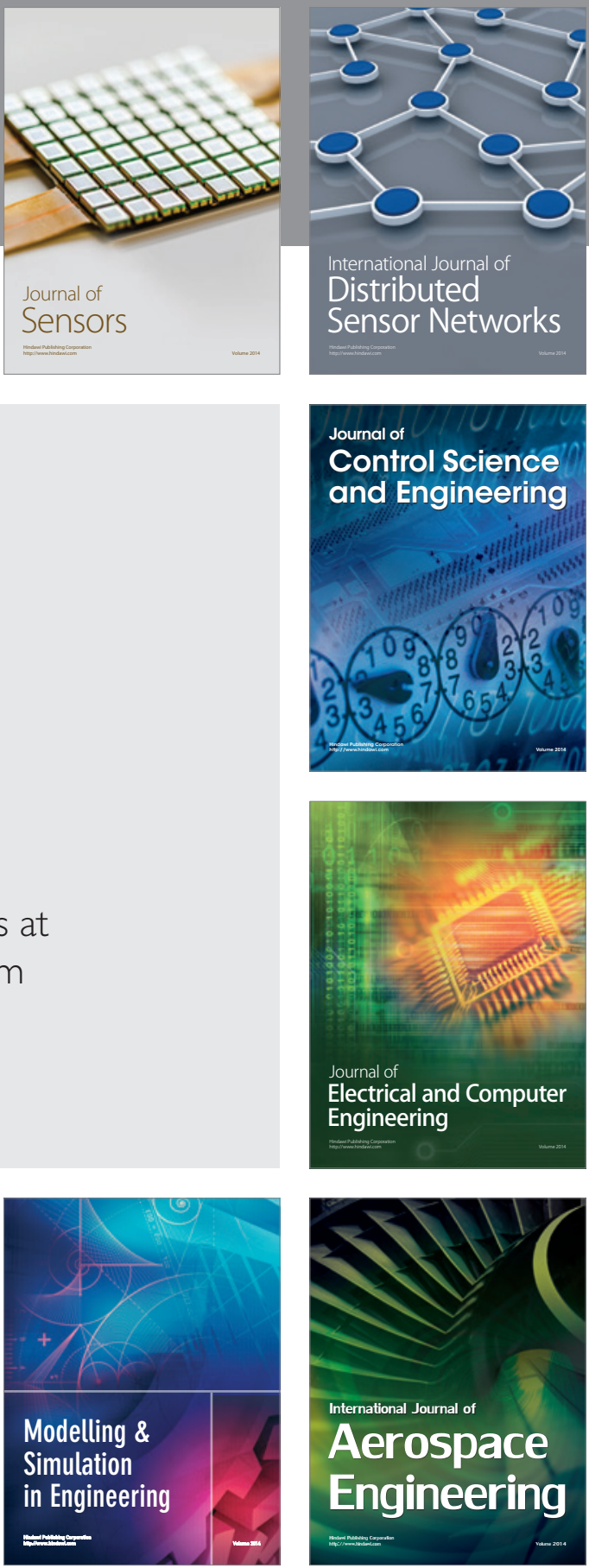

Journal of

Control Science

and Engineering
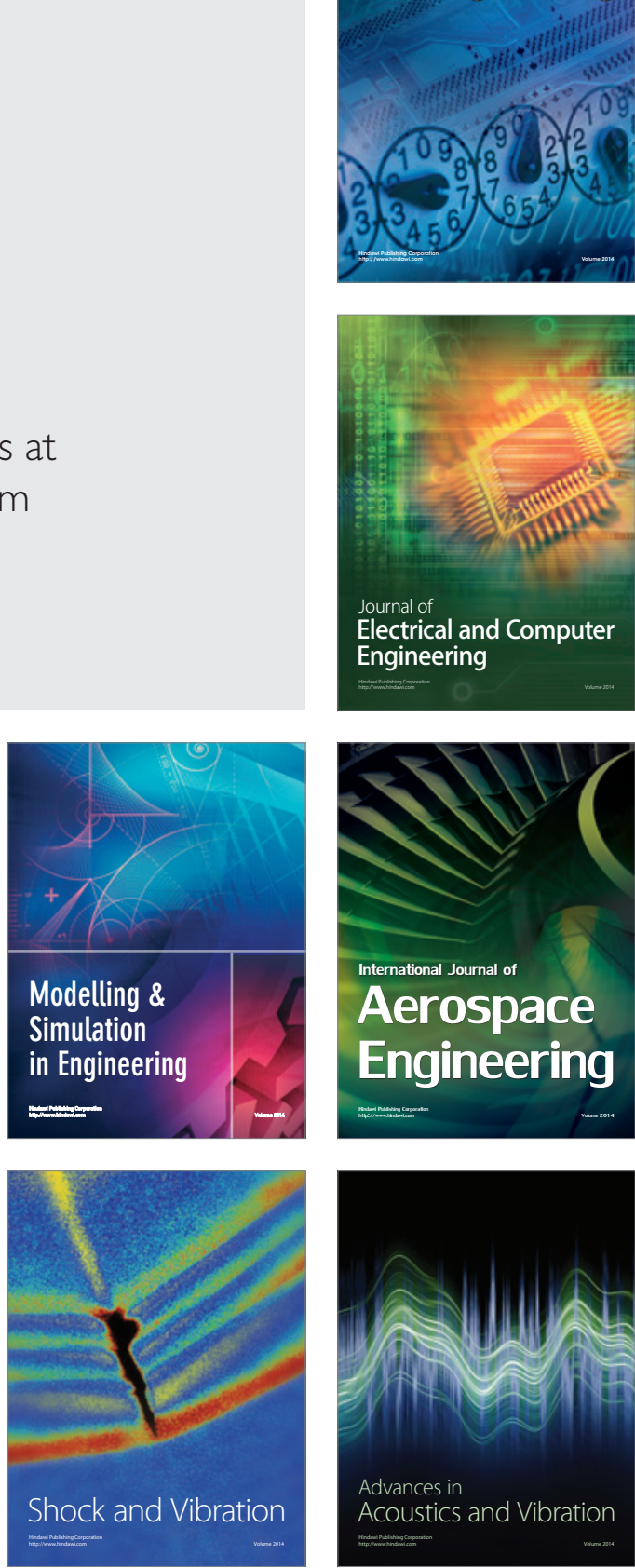